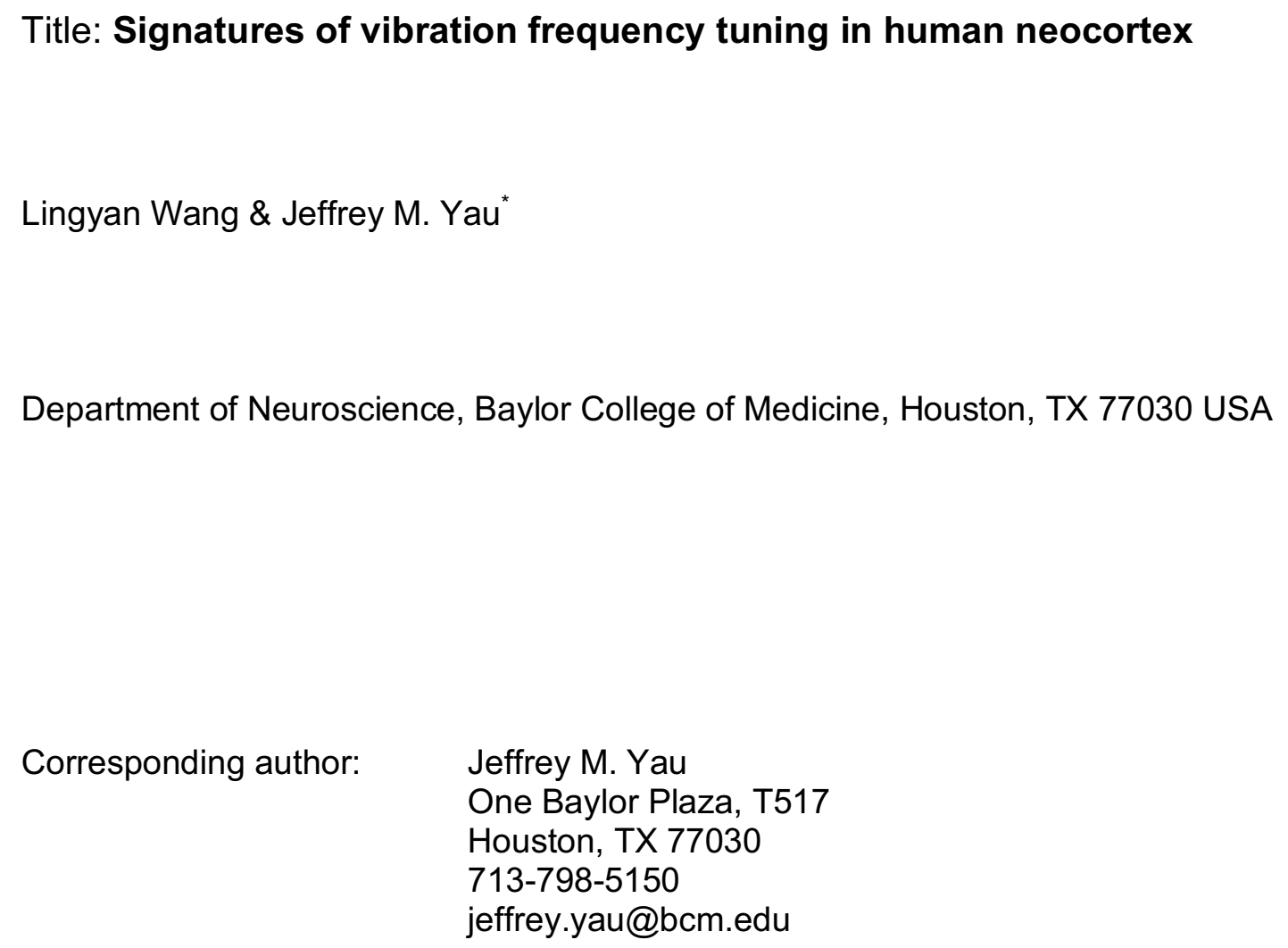




\section{ABSTRACT}

53 The spectral content of vibrations produced in the skin conveys essential information about

54 textures and underlies sensing through hand-held tools. Humans can perceive and discriminate

55 vibration frequency, yet the central representation of this fundamental feature is unknown. Using

$56 \mathrm{fMRI}$, we discovered that cortical responses are tuned for vibration frequency. Voxel tuning was

57 biased in a manner that reflects perceptual sensitivity and the response profile of the Pacinian

58 afferent system. These results imply the existence of tuned populations that may encode

59 naturalistic vibrations according to their constituent spectra.

60

61

62

63

64

65

66

67

68

69

70

71

72

73

74

75

76

77

78

79

80

81

82

83

84

85 


\section{INTRODUCTION}

87 Our physical interactions with the environment produce complex, high frequency $(>85 \mathrm{~Hz})$ vibrations in the skin whose spectral content underlie the manual perception of surface textures (Bensmaia \& Hollins, 2005; Manfredi et al., 2014) and support sensing through hand-held tools (Brisben, Hsiao, \& Johnson, 1999; Miller et al., 2018). Vibration frequency, like sound pitch, is a fundamental feature that we perceive and discriminate (Bolanowski, Gescheider, Verrillo, \& Checkosky, 1988; Convento, Rahman, \& Yau, 2018; Mountcastle, Talbot, Sakata, \& Hyvärinen, 1969). Yet evidence for frequency-tuned somatosensory circuits remains conspicuously absent, in stark contrast to the tuning observed throughout the auditory neuraxis (Hudspeth, 2014; Saal, Wang, \& Bensmaia, 2016; Wang, 2007). In human and non-human primates, vibration frequency is encoded in the periodicity of spiking activity of untuned cells in the peripheral afferent system (Johansson, Landstrom, \& Lundstrom, 1982; Talbot, Darian-Smith, Kornhuber, $\&$ Mountcastle, 1968) and the earliest cortical processing stages (Harvey, Saal, Dammann 3rd, \& Bensmaia, 2013; Lebedev \& Nelson, 1996; Mountcastle et al., 1969). Conceivably, this temporal coding of vibration frequency gives rise to a rate-based representation in tuned populations, as seen in the auditory system (Saal et al., 2016; Wang, 2007). However, frequency-tuned somatosensory neurons have never been reported in primates and tuned cells were only recently discovered in the mouse somatosensory cortex (Prsa, Morandell, Cuenu, \& Huber, 2019). The failure to establish frequency tuning in the primate brain may have been due to limited sampling of cortical territories or restricted exploration of vibrotactile stimulus space.

To search for vibration frequency tuning in the human brain, we performed whole brain functional magnetic resonance imaging ( $\mathrm{fMRI}$ ) as participants experienced a battery of vibrations on their hands while engaging in an attention-demanding frequency monitoring task (Supplementary Fig. 1). Vibrations, which were matched in perceived intensity, varied in frequency from 100 to $340 \mathrm{~Hz}$ (Supplementary Fig. 2). We characterized voxel-level responses

112 which reveal systematic tuning for vibration frequency. We compared voxel-tuning properties

113 across participants and observed consistent tuning preferences that mirrored perceptual

114 sensitivity and the response profile of the Pacinian afferent system. Lastly, we implemented an 115 encoding model to provide an account for how voxel-level frequency tuning can relate to neural 116 population responses.

\section{RESULTS}


We first defined brain regions whose blood oxygen level-dependent (BOLD) activity was modulated by vibration stimulation applied to the left or right hands (Fig. 1a; Supplementary

121 Fig. 3) irrespective of vibration frequency. Response modulation associated with right hand

122 stimulation was greater in strength $(t(6)=2.48, P=0.048)$ and more prevalent $(t(6)=4.21, P=$

$1230.0056)$ compared to left hand responses. In both hemispheres of each participant, voxel

124 responses were significantly modulated by vibrations delivered to the contralateral or ipsilateral

125 hands (F-statistic: contralateral: $7.67 \pm 0.86$; ipsilateral: $7.37 \pm 0.75)$. Response modulation

126 associated with the contralateral and ipsilateral hands was similar in strength $(t(6)=2.28, P=$

$1270.063)$ and prevalence $(t(6)=1.12, P=0.30)$.

a
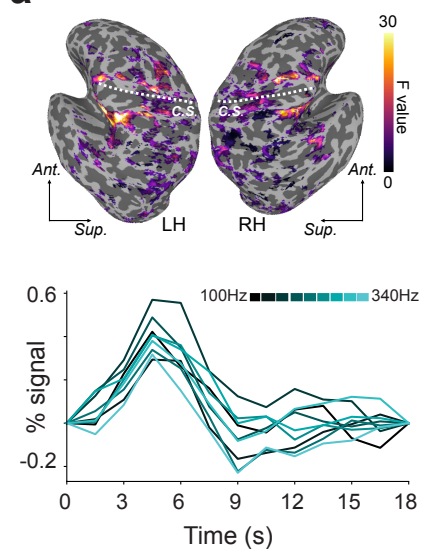

b

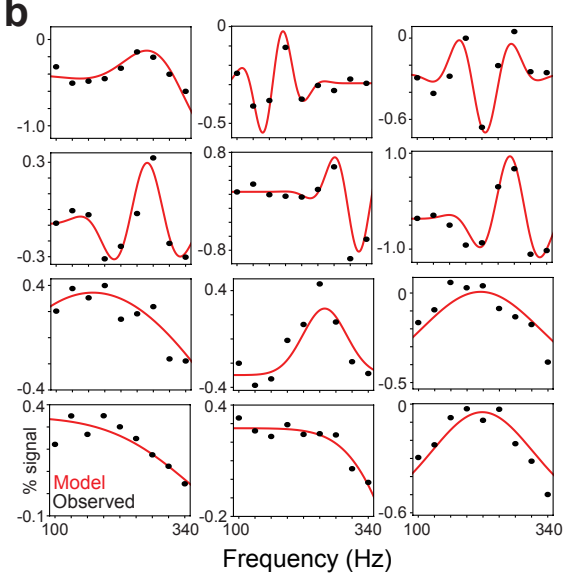

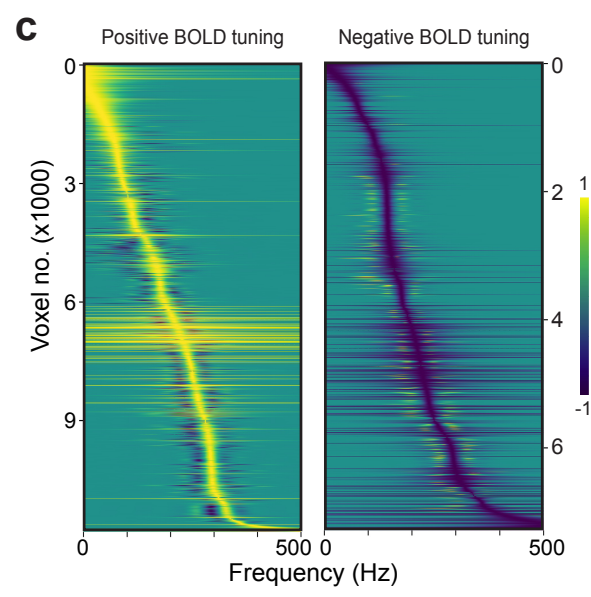

Figure 1. Frequency tuning of cortical responses to vibrations. (a) Vibrations delivered to the left or right hand are associated with significant BOLD signal modulation in an example participant's sensorimotor cortex. Dashed line indicates the central sulcus (c.s.) in the left hemisphere (LH) and right hemisphere (RH). BOLD signal time courses of an example voxel to different vibration frequencies follow stereotypical hemodynamic response profiles. Ant, anterior; Sup, superior. (b) Frequency tuning curves of example voxels (black dots indicate observed responses; red traces indicate fitted tuning functions). Frequency response patterns are consistent with Gabor tuning (top rows) or Gaussian tuning (bottom rows). (c) Normalized tuning curves for all frequency-selective voxels in example participant sorted by best modulating frequency (BF). Positive BOLD tuning voxels (left) exhibit signal increases at the BF while negative BOLD tuning voxels (right) exhibit signal decreases at the BF.

129 To characterize frequency-dependent modulation in vibration-responsive voxels (Fig. 1b), we

130 fitted voxel-level response profiles with tuning functions (Materials and Methods). Tuning along

131 a single dimension like temporal frequency can be modeled by fitting responses with simple

132 Gaussian filters that parameterize the best modulating frequency (BF) and tuning sharpness.

133 More complex frequency preferences can be modeled using Gabor filters that capture tuning 134 profiles characterized by multiple modulation fields. Across participants, $59 \pm 6.3 \%$ of vibrationresponsive voxels exhibited significant tuning (FDR-corrected $q<0.05$ ) that was described by the Gaussian model ( $r=0.67 \pm 0.013$; range: $0.23-0.97)$ or Gabor model $(r=0.57 \pm 0.025$; 
137 range: 0.36-0.95). Tuned voxels were predominantly found in parietal and frontal cortex

138 (Supplementary Fig. 4). We performed model selection for each tuned voxel (Materials and

139 Methods) and found that voxel profiles tended to be more consistent with Gaussian tuning

140 rather than Gabor tuning (proportion of voxels consistent with Gabor tuning $=0.40 \pm 0.12$ ). We

141 compared the prevalence of Gaussian vs Gabor tuning in different sensorimotor regions under

142 the assumption that simpler tuning could define primary sensory areas while more complex

143 tuning could be confined to higher-order areas. Across regions, we observed similar proportions

144 of tuned responses best described by the Gaussian and Gabor models (Supplementary Fig.

145 5). That voxels characterized by Gaussian- and Gabor-shaped tuning are interspersed in

146 parietal and frontal brain regions is inconsistent with the notion that simple frequency selectivity

147 gives way to more complex tuning over a somatosensory cortical hierarchy.

The number of tuned voxels in the left hemisphere $(5685 \pm 3353)$ and right hemisphere $(5961 \pm$ 3635 ) did not differ significantly $(t(6)=1.35, P=0.23$ ) (Supplementary Table 1). While most tuned voxels were selective for only one hand, voxels tuned to contralateral and ipsilateral stimulation were observed in both hemispheres, and $20.59 \pm 6.06 \%$ of tuned voxels were selective for vibrations applied to either hand (Supplementary Table 2). Because contralateral and ipsilateral stimulation has been associated with BOLD signal increases and decreases (Schäfer et al., 2012), respectively, we tested whether tuned voxels were more likely to exhibit negative BOLD signal changes with ipsilateral stimulation. Voxels exhibited signal increases and decreases (Fig. 1b), but the likelihood for tuned voxels to deactivate at their BF did not differ between contralateral and ipsilateral stimulation across participants $(t(6)=1.08, P=0.32$ ) or within each participant (z-statistic $=-0.028-0.23, P=0.81-0.99)$. For tuned voxels with positive or negative activity changes, frequency response profiles spanned the entire range of tested frequencies (Fig. 1c). These results imply the existence of cortical feature detectors that are selective for the frequency components comprising naturalistic vibrations (Manfredi et al., 2014).

Having established voxel-level frequency tuning, we asked whether voxel preferences were biased to frequencies near $250 \mathrm{~Hz}$, the range corresponding to the maximum response

167 sensitivity of the Pacinian afferent system (Bell, Bolanowski, \& Holmes, 1994; Bolanowski \&

168 Verrillo, 1982; Johansson et al., 1982) and the peak perceptual sensitivity in humans

169 (Bolanowski et al., 1988; Bolanowski \& Verrillo, 1982). In each participant, BF distributions (Fig.

170 2a) differed significantly from uniform (1-sample Kolmogoroz-Smirnov test; all $P<1 \mathrm{e}-15$ ) with 
171 more voxels preferring intermediate frequencies (BF: $222 \pm 22 \mathrm{~Hz}$; range: 187-258 Hz)

172 compared to lower and higher frequencies (Supplementary Table 3). We additionally tested

173 whether voxels tuned for both hands had similar frequency preferences for the left and right

174 hands (Fig. 2b), but BF values were uncorrelated between hands $(r=-0.021 \pm 0.088 ; t(6)=-$

$1750.58, P=0.58)$. The finding that cortical frequency representations, which are maintained

176 independently for the left and right hands, mirror the sensitivity profile of human observers and

177 of the peripheral afferent system is consistent with efficient coding theory (Barlow, 1961).
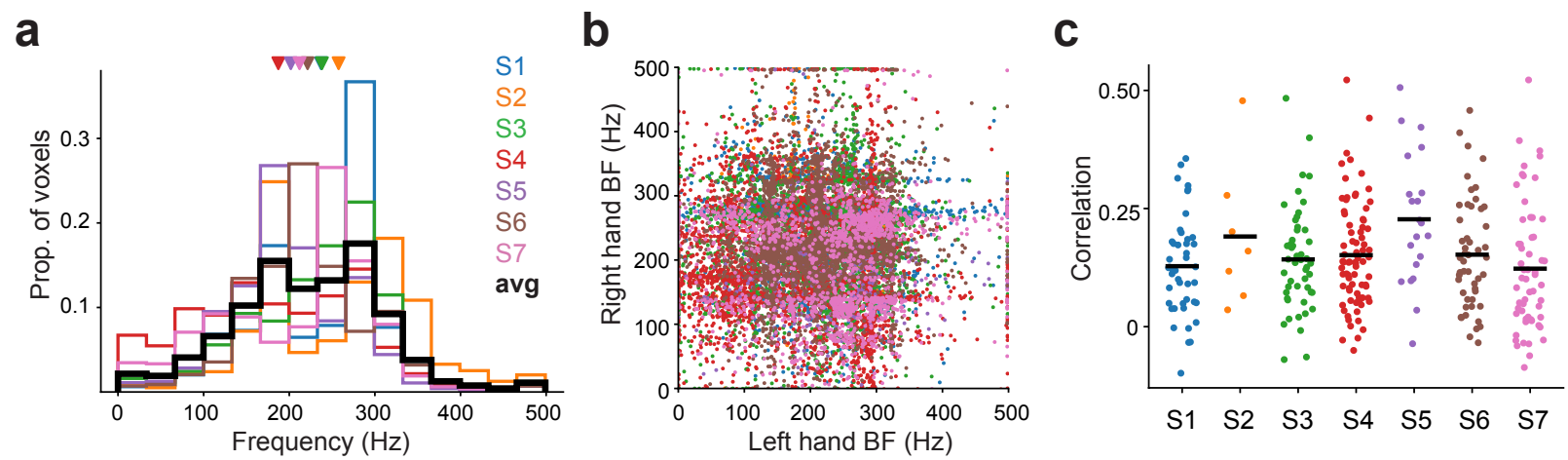

Figure 2. Best modulating frequency distributions within participants, between hands, and across activation clusters. (a) Distribution of best modulating frequency (BF) in each participant $(N=7)$. Colors indicate individual participants. Average distribution is denoted in black. Triangles indicate mean BF in each participant. (b) Relationship between left hand BF and right hand BF in voxels tuned for both hands. Frequency preferences were uncorrelated over hands (mean $r=-0.021 \pm 0.088 ; t(6)=-0.58, P=0.58$ ). (c) Relationship between voxel locations and frequency preferences. Dots indicate correlation between the physical distances separating voxel pairs within an activation cluster and their BF differences. The average correlation for each participant is denoted by the black bar. Although correlations were generally positive, BF maps were unstructured and inconsistent with tonotopic organization.

179 In the auditory cortical system, the spatial clustering of neurons with similar frequency preferences produces orderly tonotopic maps that are resolvable with fMRI (Barton, Venezia, Saberi, Hickok, \& Brewer, 2012; Martino et al., 2015). We wondered whether an analogous topography, based on vibration frequency tuning, exists in the somatosensory cortical system. We first tested if the spatial proximity between pairs of frequency-tuned voxels within activation clusters related to the similarity of their frequency preferences (Materials and Methods). The physical distances between voxels were correlated with their BF differences (Fig. 2c) for left hand responses $(r: 0.18 \pm 0.038 ; t(6)=11.30, P=2.87 \mathrm{e}-5)$ and right hand responses $(r: 0.14 \pm$ $0.042 ; t(6)=8.41, P=0.00015)$, implying that voxels with similar preferences tended to aggregate. This aggregation alone, however, is insufficient evidence for tonotopic organization because neighboring voxels could share frequency preferences simply due to spatial smoothing 
191 2007). Indeed, BF maps in each participant were generally disordered and lacked global

192 structure (Supplementary Fig. 4). We further evaluated BF maps using a more conservative

193 tonotopy definition that assumed frequency preferences within an activation cluster would be

194 arranged in a gradient over the cortical surface (Materials and Methods). A mere $0.60 \%$ of the

195 total activation clusters (2 out of 336 over all participants) comprised voxels with BFs spanning

196 the full frequency range that were arranged in a gradient. The weak evidence for orderly

197 tonotopic maps implies that frequency tuning does not define somatosensory cortical

198 topography.

Because participants were all right-hand dominant (Edinburgh handedness scores: $87 \pm 3.6$ ) and they selectively attended to vibrations delivered to their right hands during the scans, we reasoned that response profiles may differ between hands. Such differences would presumably be reflected in the distributions of tuning model parameters, which were highly consistent across participants (Supplementary Fig. 6). Indeed, we observed greater response modulation with right hand responses (Supplementary Fig. 6a) $(t(6)=2.47, P=0.048$ ), although baseline activity levels were equivalent over the hands (Supplementary Fig. 6b) $(t(6)=1.63, P=0.16$ ). We predicted that right hand responses would be more frequency selective, but tuning widths did not differ between the hands (Supplementary Fig. 6c) $(t(6)=1.94, P=0.10$ ). For voxels best described by the Gabor model, we evaluated phase parameter distributions and found that phase distributions differed between hands in all participants (Watson's two-sample test of

211 homogeneity; $\left.U^{2}=0.82-5.66, P<0.001\right)$. Despite these differences, phase distributions were

212 typically bimodal (Rayleigh test, $P<1 \mathrm{e}-15$ ) with prominent peaks at $0.5 \pi$ and $1.5 \pi$ that indicate

213 a general tendency for tuning functions to comprise balanced positive and negative peaks

214 (Supplementary Fig. 6d). Altogether, these analyses highlight the consistency of tuning 215 patterns across participants and reveal differences between left and right hand tuning profiles

216 that may be related to hand dominance or attention.

218 How might voxel-level tuning be related to cortical population activity? Vibrations delivered to

219 the glabrous skin entrains the activity in some cortical populations and frequency could be 220 represented by a spike timing code using these untuned but phase-locking neurons (Harvey et

221 al., 2013; Lebedev \& Nelson, 1996; Mountcastle et al., 1969). However, the frequency-response

222 profiles of these neurons - characterized by spike rates that increase monotonically with

223 frequency - are incompatible with voxel-level Gaussian and Gabor tuning, assuming the BOLD

224 signal reflects aggregate population activity (Klink, Chen, Vanduffel, \& Roelfsema, n.d.). 
a
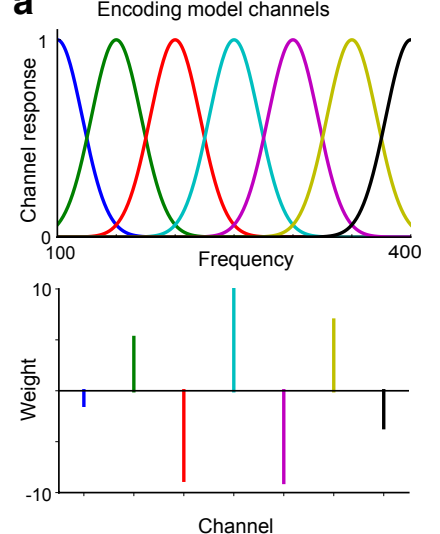

d b
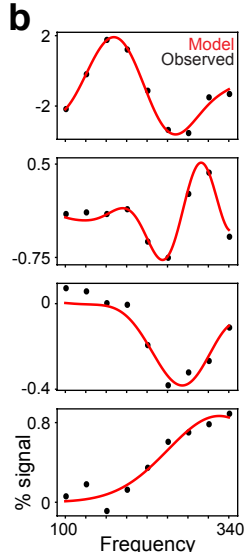
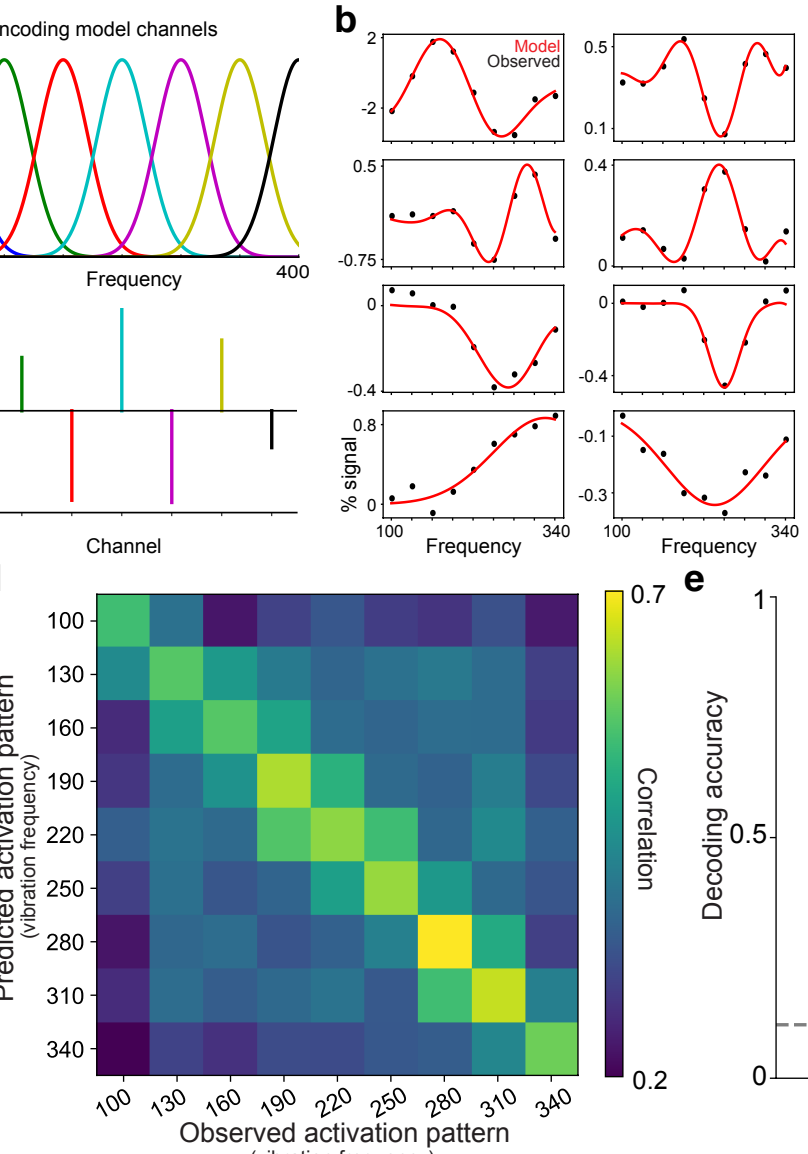

C

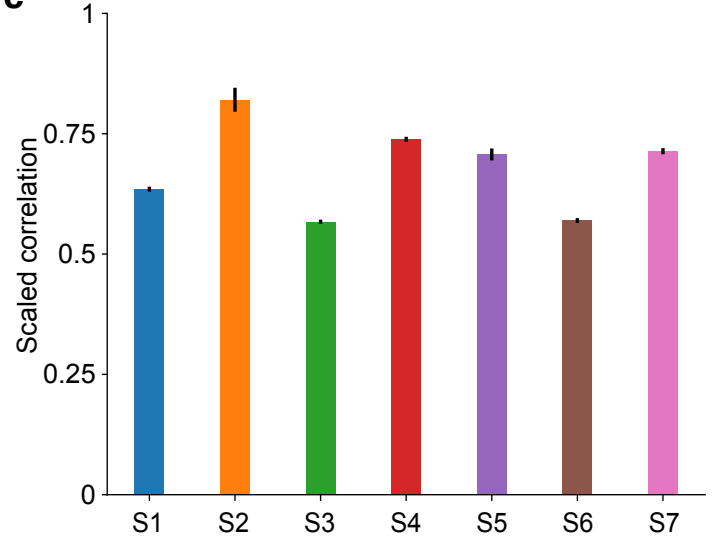

e 11

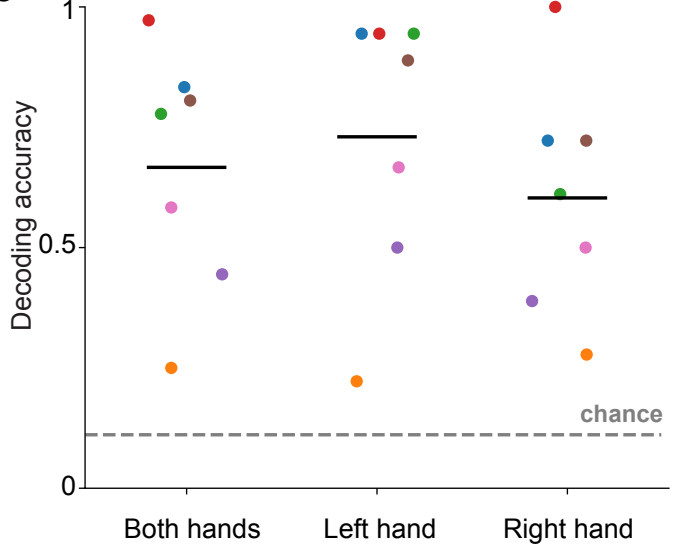

(vibration frequency)

Figure 3. Encoding model based on activity of putative frequency-tuned neural populations. (a) Gaussian channels represent neural populations that respond selectively to different vibration frequencies. The encoding model assumes that a voxel's response to any given vibration frequency is the weighted sum of the activity in the channels. (b) Encoding model captures tuned response patterns in example voxels (black dots). Red curves indicate model-predicted responses profiles. (c) Bars indicate voxel-averaged scaled model performance within each participant. The model is trained on one fold of data and tested on a held-out fold. Model performance is the correlation between the model predictions and the test data, normalized by the correlation between the two folds of data (which represent the maximum correlation possible given the noise in the data). Error bar indicate s.e.m. (d) Correlation matrix indicates the similarity between multivoxel activation patterns predicted by the encoding model and observed patterns in the held-out data. Correlations are averaged over hands and participants. For decoding, an algorithm identifies the model-predicted pattern yielding the highest correlation with an observed pattern to infer the frequency condition. (e) Cross-validated decoding performance for both hands and each hand separately. Black line indicates group averaged accuracy. Colored dots indicate individual participants. Dashed line indicates chance performance.

225 Alternatively, vibration frequency could be carried in the activity of tuned populations, which

226 have recently been identified in mouse somatosensory cortex (Prsa et al., 2019). Phase-locking

227 responses are less prominent as one ascends the cortical hierarchy (Harvey et al., 2013), which

228 may reflect a transition to a rate-based code. As a proof of concept, we implemented an

229 encoding model to explore how the activity of tuned populations could relate to voxel-level

230 responses (Materials and Methods). We assumed that a voxel's response reflects the weighted 
231 combination of activity in neural populations selective for different frequencies (Fig. 3a). These

232 encoding models recapitulated observed voxel profiles (Fig. 3b) and accounted for substantial

233 response variance (Fig. 3c; scaled goodness-of-fit: $0.68 \pm 0.086$ ). We also verified that the

234 encoding models captured voxel tuning by performing a decoding analysis (Materials and

235 Methods). The models predicted multivoxel activity patterns that closely resembled observed

236 patterns (Fig. 3d). Accordingly, a simple decoder (Fig. 3e) identified the frequencies associated

237 with different measured patterns with an accuracy $(67 \% \pm 23 \%)$ far exceeding chance

238 performance $(11 \%)(t(6)=5.82, P<0.0011)$. Lastly, we considered how phase-locking

239 populations could contribute to voxel responses and found that voxel tuning could be

240 recapitulated only if the phase-locking neurons exhibited some degree of frequency selectivity

241 (Supplementary Fig. 7). These modeling results confirm that frequency information is carried in

242 voxel responses and provide a conceptual framework for relating voxel tuning to frequency-

243 selective neural populations.

\section{DISCUSSION}

246 We find that voxel-level BOLD signals are modulated by vibrations in a manner that clearly 247 reflects frequency selectivity. Voxel tuning spans the range of frequencies that are relevant for

248 fine texture perception (Bensmaia \& Hollins, 2005; Manfredi et al., 2014). Frequency

249 preferences are consistent across individuals and systematic with a greater share of voxels

250 preferring frequencies that optimally drive the Pacinian afferent system (Bell et al., 1994;

251 Bolanowski \& Verrillo, 1982; Johansson et al., 1982). This cortical bias may underlie our

252 enhanced perceptual sensitivity for vibrations near $250 \mathrm{~Hz}$ (Bolanowski et al., 1988; Bolanowski

253 \& Verrillo, 1982). Our finding that cortical representations mirror environmental statistics,

254 peripheral afferent profiles, and perceptual sensitivity is consistent with the predictions of

255 efficient coding theory (Barlow, 1961).

257 Conceivably, frequency-tuned voxel activity reflects neurons in the primate somatosensory

258 cortical system that are analogous to vibration selective cells recently identified in mice.

259 Importantly, although individual neurons in mouse somatosensory cortex exhibit vibration tuning

260 (Prsa et al., 2019), such neurons have never been reported in the primate brain. Limited

261 sampling of cortical populations and territories may have obscured the presence of frequency-

262 tuned neurons. Alternatively, frequency tuning may be a property that only emerges at a

263 population level in primates. Arbitrating between these possibilities will require large scale

264 neurophysiological recordings, which can be guided by our neuroimaging findings. 
Future studies will also need to address the mechanisms that generate frequency selectivity in the somatosensory system: Our data reveal frequency selective cortical responses despite the absence of fine tuning in peripheral and subcortical processing stages. This contrasts with the auditory system, where frequency tuning exists throughout the neuraxis, even at the receptor level (Hudspeth, 2014). Cortical tuning may reflect the central convergence of submodality signals that are initially carried by distinct populations in the peripheral afferent system (Pei,

272 Denchev, Hsiao, Craig, \& Bensmaia, 2009; Saal \& Bensmaia, 2014; Saal, Harvey, \& Bensmaia,

273 2015). In fact, recent evidence has challenged the traditional functional dichotomy between

274 Pacinian and non-Pacinian perceptual channels by positing a universal frequency decoding

275 system (Birznieks et al., 2019). Beyond submodality convergence, varying distributions of

276 excitatory and inhibitory neurons may also underlie the diversity of frequency-selective

277 population responses across sensory cortex (Hughes et al., 2021). At a cellular level, short term

278 synaptic depression may impose a frequency dependent filter on information transmission

279 (Rosenbaum, Rubin, \& Doiron, 2012) and mediate the conversion from temporal coding to rate coding (Lee, Wang, \& Bendor, 2020).

Regardless of the mechanism, our data reveal somatosensory cortical activity in human neocortex that is tuned for vibration frequency. Analogous frequency encoding schemes in the somatosensory and auditory systems may facilitate the extensive crosstalk between touch and audition in the temporal frequency domain (Crommett, Madala, \& Yau, 2019; Crommett, PerezBellido, \& Yau, 2017; Yau, Olenczak, Dammann, \& Bensmaia, 2009; Yau, Weber, \& Bensmaia, 2010). Moreover, frequency-selective cortical filters offer an efficient scheme for representing the complex spectra of vibrations encountered in naturalistic touch.

\section{MATERIALS AND METHODS}

291 Participants. Seven healthy adult volunteers (5 females; mean age \pm SD: $26 \pm 2.8$ years; aged 292 20-29 years) participated in the study. All participants were right-handed (Oldfield, 1971) 293 (Laterality quotient: $87.1 \pm 9.7$ ). The sample size was set on the basis that any significant and 294 consistent outcomes established in 7 out of 7 subjects would be statistically generalizable 295 according to a 2-tail binomial test $(P<0.05)$. Participants had normal or corrected-to-normal 296 vision. Testing procedures were approved by the Baylor College of Medicine Institutional Review

297 Board. All participants provided written consent and were paid for their participation or waived 298 payment. 
MRI acquisition. All scans were conducted in the Core for Advanced MRI (CAMRI) at Baylor College of Medicine. MRI data were acquired on a 3-Tesla MAGNETOM Trio scanner with Prisma fit (Siemens, Erlangen, Germany) using a 64-channel head coil. Anatomical data were acquired using a T1-weighted magnetization prepared rapid acquisition gradient echo sequence (MPRAGE; TR = $2300 \mathrm{~ms} ;$ TE $=2.98 \mathrm{~ms}$; flip angle $=9^{\circ} ; 1 \mathrm{~mm}^{3}$ voxels). Functional data were obtained using an axial echo-planar imaging (EPI) sequence with simultaneous multi-slice (SMS) acceleration (TR = $1500 \mathrm{~ms} ; \mathrm{TE}=33 \mathrm{~ms}$; flip angle $=90^{\circ}$; GRAPPA factor $=2$; SMS factor = 3; FOV = $192 \mathrm{~mm}$; 69 slices; $2 \mathrm{~mm}^{3}$ voxels; 380 volumes per scan) that covered all of the cortical volume and part of the cerebellum. Each participant underwent 12 functional scans ( 9.5min/scan) divided across 2 sessions ( $5.9+/-7.4$ days inter-session interval).

Tactile stimulation. Vibrotactile cues were delivered to the distal pad of the participant's left and right index fingers using an MRI-compatible piezoelectric tactor (Engineering Acoustics, Inc., Casselbery, FL). Tactors were fastened to the distal finger pads with self-adherent cohesive wrap bandages. Tactors were controlled using the EAI Tactor Development Kit and stimulus timing was determined using custom Matlab scripts. The vibration set comprised 9 frequencies: 100, 130, 160, 190, 220, 250, 280, 310, and 340Hz. Vibrations were matched in perceived intensity with amplitudes (gain: 71.4-97.4 arbitrary units according to EAI controller) determined in preliminary behavioral experiments using the method of adjustment. To further ensure that participants attended to vibration frequency rather than intensity during the scans, we applied a random $\pm 5 \%$ jitter in amplitude on each stimulus presentation. Offline, we measured vibration amplitudes (unloaded) using a laser displacement sensor (ZX2-LD50, Omron, Hoffman Estates, IL) (displacement range: 0.414-0.504mm) and confirmed tactor reliability (Supplementary Fig. 2).

Frequency monitoring task and scans. Participants were scanned in an event-related design as they performed a vibration frequency monitoring (oddball detection) task (Perez-Bellido, Barnes, Crommett, \& Yau, 2017) while maintaining visual fixation. Each scan comprised unimanual and bimanual events. An event comprised a series of 3 vibration stimuli (stimulus duration: $700 \mathrm{~ms}$; inter-stimulus interval: $300 \mathrm{~ms}$ ). On the majority of events (regular events; 66/76 in each scan corresponding to 2 repetitions each of 9 right hand frequencies, 9 left hand frequencies, and 15 bimanual frequency combinations), the frequency of the three vibrations was identical. All of the analyses included in this report were based on the unimanual regular events. 
333 On a subset of events (oddball events; 10/76 in each scan), the frequency of the second vibration

334 differed from the first and third vibrations in the series (frequency difference: 120-240Hz).

335 Participants were instructed to report the occurrence of oddball events using a foot pedal

336 response (Current Designs, Philadelphia, PA). Reliable detection of oddball events

337 (Supplementary Fig. 1) indicated that participants attended to vibration frequency. The

338 responding foot was counter-balanced across sessions over participants. To control for spatial

339 attention effects, oddball events only occurred on the right hand (on unimanual and bimanual

340 events) such that attention was directed toward each subject's dominant hand throughout the

341 scan. Events were separated by 3, 4.5, 6, or 7.5-s intervals with order and timing determined

342 pseudo-randomly using Optseq2 (http://surfer.nmr.mgh.harvard.edu/optseq).

Behavioral analysis. Given the unequal number of oddball and regular events, we quantified oddball detection performance by computing an $\mathrm{F}_{1}$ score for each subject (Powers, 2011):

$$
\mathrm{F}_{1}=2 * \frac{(\text { Precision } * \text { Recall })}{\text { Precision }+ \text { Recall }}
$$

where Precision is defined as the number of hits (correctly detected oddball events) divided by the sum of hits and false alarms (events incorrectly identified as oddball) and Recall is defined as the number of hits divided by the sum of hits and misses (oddball events not detected). $F_{1}$ scores range from 0-1 with higher scores indicating better performance. For each subject, we determined if the observed $F_{1}$ score was greater than that expected by chance (Supplementary Fig. 1) by generating a null distribution of $F_{1}$ scores assuming the observed number of positive responses with shuffled event labels over 1000 permutations.

fMRI analyses. Data preprocessing and first-level analyses were performed using AFNI (Cox, 1996). Each participant's data were preprocessed using standard procedures (afni_proc.py) including: (i) slice timing correction (3dTshift); (ii) image co-registration (align_epi_anat.py); (iii) 360 functional image alignment (3dvolreg); (iv) spatial blurring with a 4-mm FWHM filter (3dmerge); 361 (v) mean-normalization of each voxel's signal (3dcalc). Preprocessed voxel-wise data were 362 modeled using multiple linear regression (3dDeconvolve): general linear models (GLM) 363 comprised 34 regressors corresponding to left hand stimulation (9 frequencies), right hand 364 stimulation (9 frequencies), 15 bimanual conditions, and oddballs. Each regressor was created 365 using a gamma-variate convolution kernel. The GLM comprised head motion and drift parameters 
as nuisance regressors. GLM coefficients were taken as the voxel response associated with each condition. A single GLM was fitted to the whole 12-scan dataset to define the analysis mask comprising voxels whose activity was modulated by either left hand or right hand stimulation. For the tuning models and encoding model analyses, separate GLMs were fitted after dividing the full dataset into 2 folds corresponding to the 6 scans from each scanning session. Unless otherwise noted, analyses were performed in native volume space. For displaying purposes, each

372 participant's data were projected into surface space. Surface models were constructed from each

373 participant's anatomical scans using Freesurfer (Dale, Fischl, \& Sereno, 1999). The analysis

374 exploring the topographic organization of frequency preferences was performed in native surface 375 space.

In each participant, we defined an analysis mask by identifying voxels whose activity was modulated by either left hand or right hand stimulation. For each hand separately, an omnibus Fstatistic was computed to quantify the significance of each voxel's responses to the 9 vibration frequencies. The full analysis mask was the union of the left hand and right hand F-statistic maps, thresholded at a false discovery rate (FDR) corrected $q<1 \mathrm{e}-4$ over the whole brain.

Vibration frequency tuning functions. To test for vibration frequency tuning, we fitted parametric tuning functions to each voxel's frequency response profiles estimated from the 2 data folds. If a voxel's response profiles were inconsistent over the folds, a tuning model fit to these data would be meaningless. Accordingly, we only fit tuning functions to voxels whose across-fold Pearson correlation exceeded 0.2. For voxels with consistent profiles across folds, we fitted simple and complex tuning functions and performed model competition to determine the model most appropriate for each voxel given its complexity and performance. Models were fitted to each voxel's response data using the method of least squares which minimized the error between observed and predicted data. Responses to left and right hand stimulation were considered separately. by a Gaussian function:

$$
r=A e^{\frac{-0.5(f-\mu)^{2}}{\sigma^{2}}}+b
$$


where $r$ is the predicted voxel response to a vibration with frequency $f, A$ is a gain term, $\mu$ is the best modulating frequency, $\sigma$ is the tuning width, and $b$ indicates the baseline activity level over all frequencies. The Gaussian model comprised 4 free parameters.

To capture more complex frequency tuning patterns, we assumed a voxel's response profile was characterized by a Gabor function, a cosine wave modulated by a Gaussian window:

$$
r=A e^{\frac{-0.5(f-\mu)^{2}}{\sigma^{2}}} \cos \left(\frac{2 \pi}{\lambda}(f-\mu)+\phi\right)+b
$$

where $r$ is the predicted voxel response to a vibration with frequency $f, A$ is a gain term, $\mu$ is the center of the Gaussian, $\sigma$ is the spread of the Gaussian, $\lambda$ and $\phi$ are the wavelength and phase of the wave, and $b$ indicates the baseline activity level over all frequencies. The Gabor model comprised 6 free parameters. In preliminary analysis, we found that estimating $\lambda$ with no constraints could yield small wavelength values that reflected the noise in the data. Accordingly, we constrained $\lambda$ by requiring the $\lambda / \sigma$ ratio to be $>2.25$ in the final analysis.

To determine whether a voxel's responses were better captured by the Gaussian or Gabor models, we compared models using Akaike information criterion (AIC) (Burnham \& Anderson, 417 2004):

$$
A I C=n * \ln (R S S)+2 k
$$

where $n$ is the number of data points used to fit the models, RSS is the residual sum of squared errors, and $k$ is the number of free parameters. The AIC-preferred model of each voxel was taken as that which yielded the smaller AIC value. We then computed the correlation between the AIC-preferred model predictions and the observed data to quantify goodness-of-fit. Voxels were considered to be tuned if the correlation between model predictions and observed data was statistically significant after correcting for the number of modeled voxels (FDR corrected $q<$ 0.05 using the Benjamini-Hochberg procedure). defined the peak of a tuning function as the curve portion corresponding to the greatest

431 (modulus) response modulation. Best modulating frequency (BF; $1-500 \mathrm{~Hz}$ ) was the $\mu$ 
432 parameter for Gaussian models or the frequency corresponding to the peak for Gabor models.

433 The gain term indicated the (unsigned) magnitude of response modulation. The baseline

434 parameter represented basal activity common to all frequencies. Tuning sign (positive or

435 negative) corresponded to direction of activity change relative to the baseline level at the peak.

436 The full width at half maximum (FWHM) along the peak indicated the tuning selectivity of each

437 voxel.

439 Voxel-wise encoding models. We implemented a simple channel encoding model to predict 440 voxel-level activity by assuming the existence of frequency-tuned cortical neurons like those 441 recently identified in mouse somatosensory cortex (Prsa et al., 2019). We modeled the 442 normalized activity levels $(R)$ of a cortical population in response to a vibration with frequency $f$ 443 using a Gaussian channel:$$
R=e^{\frac{-(f-\mu)^{2}}{2 \sigma^{2}}}
$$

where $\mu$ is the population's BF and $\sigma$ is the channel tuning width. We assumed a voxel comprises different populations with unique frequency preferences, so the full encoding model predicted a voxel's response $(r)$ as a linear combination of activity from multiple populations:

$$
r=\sum_{i=1}^{\# c h} w_{i} R_{i}
$$

453 where $R_{i}$ is the normalized activity of the $i^{\text {th }}$ population and $w_{i}$ is a weight that describes the 454 population's contribution to the voxel's overall response. We modeled each voxel using 7 455 channels with predefined BF values. Accordingly, an encoding model was fitted to the response 456 profiles of each tuned voxel by estimating the channel weights and a tuning width parameter 457 that was shared over all the channels. Model fitting was performed using 2-fold cross-validation. 458 Parameters were estimated using the method of least squares to minimize the error between 459 model predictions and the tuning curve describing one data fold. Model performance was 460 computed as the Pearson correlation between the model predictions and the data in the second 461 fold. The final goodness-of-fit was the cross-validated model performance averaged over the 462 two folds. Because the cross-validated goodness-of-fit depends on the consistency of the two 463 folds, we normalized model performance by the across-fold correlation and report scaled 
correlations. For voxels tuned to both hands, separate models were fitted to explain left hand and right hand responses.

467 In separate analyses, we considered how voxel level activity may be related to phase-locking 468 neurons that have been identified in non-human primates (Harvey et al., 2013; Lebedev \& 469 Nelson, 1996; Mountcastle et al., 1969) (Supplementary Fig. 7). We reasoned that the total 470 spiking activity of these neurons would be minimal at low vibration frequencies and grow with 471 increases in vibration frequency. Importantly, phase-locking neurons would fail to respond on 472 every stimulus cycle at high vibration frequencies because of neural refractoriness, so 473 population firing rates would saturate. We modeled this ramp-to-plateau response profile of a neural population using a rectified linear unit (ReLU) as a channel in our encoding model:

$$
R(s)=s\left(f-f_{0}\right)
$$

where $R(s)$ is the normalized population activity to a vibration with frequency $f, s$ is a slope parameter describing the relationship between population activity and frequency, and $f_{0}$ is the lowest frequency at which the population responds (set to $1 \mathrm{~Hz}$ ). Because neural activity depends on vibration amplitude (Harvey et al., 2013) and populations can differ in their sensitivity to vibration amplitude, we modeled different populations (i.e., channels) as rectified linear units with different slopes. Note that by allowing the channels to have different slopes, we assume that neural populations in a voxel respond differentially over vibration frequencies thereby building frequency tuning into the model. The full encoding model, then, predicted a voxel's response $(r)$ as a linear combination of activity from multiple populations:

$$
r=\sum_{i=1}^{\# c h} w_{i} R\left(s_{i}\right)
$$

where $R\left(s_{i}\right)$ is the normalized activity of the $i^{\text {th }}$ population defined by slope $s_{i}$ and $w_{i}$ is a weight

491 that describes the population's contribution to the voxel's overall response. We assumed each

492 voxel comprised 8 populations with predefined slopes. The ReLU models were trained and

493 tested in the same manner as the Gaussian channel model.

495 We implemented a simple decoder using the encoding models to verify further that the models 496 captured the frequency response profiles of the voxels. The decoding analysis included only the 
voxels with significant encoding model performance $(P<0.05)$. Using the encoding models fitted to one data fold, we generated multivoxel activity patterns for each vibration frequency. These patterns served as labeled templates against which the observed multivoxel activity patterns in the other data fold could be compared. For decoding, we computed the correlations between an observed activation pattern and each of the template patterns predicted with the encoding models. The template pattern yielding the maximum correlation was taken as the decoded frequency. For each participant, decoding performance was the accuracy averaged over the two folds. Because distinct voxel sets exhibited tuning for left and right hand stimulation, the encoding and decoding analyses were performed separately for each hand.

Topography analysis. We performed two analyses to establish evidence for a topographic organization based on voxel frequency preferences. We first tested whether the physical distance (in volume space) between pairs of voxels related to the similarity of their BFs. For each participant, this analysis was performed within activation clusters (minimum cluster size = 40 voxels). For each cluster, we defined $\overrightarrow{\Delta d}$ as a vector of distances between each pair of

512 voxels and $\overrightarrow{\Delta \mathrm{BF}}$ as a vector of pairwise voxel BF differences. We computed the correlation

513 between $\overrightarrow{\Delta \mathrm{d}}$ and $\overrightarrow{\Delta \mathrm{BF}}$ for each activation cluster. At the group level, we tested whether the

514 average (within participant) correlation over clusters differed significantly from 0.

The second analysis tested whether frequency preferences within activation clusters were arranged in a gradient pattern over the cortical surface (minimum cluster size: 60 surface nodes). Two conditions needed to be met in order for an activation cluster to be considered tonotopic. First, the cluster needed to contain nodes with BFs that spanned the full frequency range. For each cluster, we binned BF values from $50-450 \mathrm{~Hz}$ in $50-\mathrm{Hz}$ steps. We only further considered clusters that had at least one node in each BF bin. Second, BF values within a

522 cluster were required to be systematically arranged. For each cluster, we defined an axis that

523 passed through the cluster's center. We then projected each node's BF onto the axis and

524 performed linear regression between the BF values and node locations along the axis. A

525 significant linear regression fit indicated that BFs were ordered in a gradient along the axis.

526 Because we were agnostic to the orientation of potential BF gradients, we defined repeated the 527 analysis along 4 axes $\left(0^{\circ}, 45^{\circ}, 90^{\circ}\right.$, and $\left.135^{\circ}\right)$ for each cluster. 
531 independent proportions test. For circular data, we performed the Rayleigh uniformity test and

532 Watson's two-sample test of homogeneity. All tests were performed using Python 3.7 or R 3.5.1.

\section{ACKNOWLEDGEMENTS}

535 This work was supported by R01NS097462 (JMY). We acknowledge BCM's Core for Advanced

$536 \mathrm{MRI}$ (CAMRI) and the Computational and Integrative Biomedical Research Center (CIBR). We

537 thank Md. Shoaibur Rahman with data collection. We thank Nuo Li, Mike Beauchamp, Yue

538 Zhang, and Meghan Robinson for helpful feedback and technical support. We thank Jing Lin for

539 help with vibrometry and James Romesberg for assistance with constructing the foot pedal. We

540 are grateful to Yau Lab members for helpful discussions.

\section{AUTHOR CONTRIBUTIONS}

543 J.M.Y. designed the experiment. L.W. collected the data. L.W. and J.M.Y. wrote the analysis code, analyzed, and interpreted the data. L.W. and J.M.Y. wrote the manuscript.

COMPETING INTERESTS

The authors declare no competing interests.

\section{REFERENCES}

Barlow, H. (1961). Possible principles underlying the transformations of sensory messages. In Rosenblith W (Ed.), Sensory communication (pp. 217-234). Cambridge, MA: MIT.

Barton, B., Venezia, J. H., Saberi, K., Hickok, G., \& Brewer, A. A. (2012). Orthogonal acoustic dimensions define auditory field maps in human cortex. Proceedings of the National Academy of Sciences, 109(50), 20738-20743. https://doi.org/10.1073/pnas.1213381109//DCSupplemental.www.pnas.org/cgi/doi/10.1073/pnas.1213381109

Bell, J., Bolanowski, S. J., \& Holmes, M. H. (1994). The structure and function of Pacinian corpuscles: A review. Progress in Neurobiology, 42, 79-128.

Bensmaia, S., \& Hollins, M. (2005). Pacinian representations of fine surface texture. Percept Psychophys, 67(5), 842-854.

Birznieks, I., Mclntyre, S., Nilsson, H. M., Nagi, S. S., Macefield, V. G., Mahns, D. A., \& Vickery, R. M. (2019). Tactile sensory channels over-ruled by frequency decoding system that utilizes spike pattern regardless of receptor type. Elife, 8:e46510.

Bolanowski, S. J., Gescheider, G. A., Verrillo, R. T., \& Checkosky, C. M. (1988). Four channels mediate the mechanical aspects of touch. Journal of the Acoustical Society of America, 84, 1680-1694.

Bolanowski, S. J., \& Verrillo, R. T. (1982). Temperature and criterion effects in a somatosensory subsystem: a neurophysiological and psychophysical study. Journal of Neurophysiology, 48, 836855.

Brisben, A. J., Hsiao, S. S., \& Johnson, K. O. (1999). Detection of vibration transmitted through an object grasped in the hand. Journal of Neurophysiology, 81, 1548-1558.

Burnham, K. P., \& Anderson, D. R. (2004). Multimodel inference: Understanding AIC and BIC in model selection. Sociological Methods and Research, 33(2), 261-304. https://doi.org/10.1177/0049124104268644

Convento, S., Rahman, M. S., \& Yau, J. M. (2018). Selective Attention Gates the Interactive Crossmodal 
Coupling between Perceptual Systems. Current Biology, 28(5), 746-752. https://doi.org/10.1016/j.cub.2018.01.021

Cox, R. W. (1996). AFNI: software for analysis and visualization of functional magnetic resonance neuroimages. Comput Biomed Res, 29(3), 162-173.

Crommett, L. E., Madala, D., \& Yau, J. M. (2019). Multisensory Perceptual Interactions Between HigherOrder Temporal Frequency Signals. Journal of Experimental Psychology: General, 148(7), 11241137.

Crommett, L. E., Perez-Bellido, A., \& Yau, J. M. (2017). Auditory adaptation improves tactile frequency perception. J Neurophysiol, 117, 1352-1362. https://doi.org/10.1152/jn.00783.2016

Dale, A. M., Fischl, B., \& Sereno, M. I. (1999). Cortical surface-based analysis. I. Segmentation and surface reconstruction. Neuroimage, 9(2), 179-194. https://doi.org/10.1006/nimg.1998.0395

Harvey, M. A., Saal, H. P., Dammann 3rd, J. F., \& Bensmaia, S. J. (2013). Multiplexing stimulus information through rate and temporal codes in primate somatosensory cortex. PLoS Biol, 11(5), e1001558. https://doi.org/10.1371/journal.pbio.1001558

Hudspeth, A. J. (2014). Integrating the active process of hair cells with cochlear function. Nature Reviews Neuroscience, 15(9), 600-614. https://doi.org/10.1038/nrn3786

Hughes, C. L., Flesher, S. N., Weiss, J. M., Boninger, M., Collinger, J. L., \& Gaunt, R. A. (2021). Perception of microstimulation frequency in human somatosensory cortex. Elife, 10:e65128.

Johansson, R. S., Landstrom, U., \& Lundstrom, R. (1982). Responses of mechanoreceptive afferent units in the glabrous skin of the human hand to sinusoidal skin displacements. Brain Research, 244, 1725.

Klink, P. C., Chen, X., Vanduffel, W., \& Roelfsema, P. R. (n.d.). Population receptive fields in non-human primates from whole-brain fMRI and large-scale neurophysiology in visual cortex. Elife.

Lebedev, M. A., \& Nelson, R. J. (1996). High-frequency vibratory sensitive neurons in monkey primary somatosensory cortex: entrained and nonentrained responses to vibration during the performance of vibratory-cued hand movements. Experimental Brain Research, 111, 313-325.

Lee, J. H., Wang, X., \& Bendor, D. (2020). The role of adaptation in generating monotonic rate codes in auditory cortex. PLoS Computational Biology, 16(2), e1007627.

Manfredi, L. R., Saal, H. P., Brown, K. J., Zielinski, M. C., Dammann 3rd, J. F., Polashock, V. S., \& Bensmaia, S. J. (2014). Natural scenes in tactile texture. J Neurophysiol, 111(9), 1792-1802. https://doi.org/10.1152/jn.00680.2013

Martino, F. De, Moerel, M., Ugurbil, K., Goebel, R., Yacoub, E., \& Formisano, E. (2015). Frequency preference and attention effects across cortical depths in the human primary auditory cortex. Proceedings of the National Academy of Sciences, 112(52), 16036-16041. https://doi.org/10.1073/pnas.1507552112

Miller, L. E., Montroni, L., Koun, E., Salemme, R., Hayward, V., \& Farnè, A. (2018). Sensing with tools extends somatosensory processing beyond the body. Nature, 561(7722), 239-242. https://doi.org/10.1038/s41586-018-0460-0

Mountcastle, V. B., Talbot, W. H., Sakata, H., \& Hyvärinen, J. (1969). Cortical neuronal mechanisms in flutter-vibration studied in unanesthetized monkeys. Neuronal periodicity and frequency discrimination. Journal of Neurophysiology, 32(3), 452-484.

Oldfield, R. C. (1971). The assessment and analysis of handedness: the Edinburgh inventory. Neuropsychologia, 9, 97-113.

Pei, Y. C., Denchev, P. V, Hsiao, S. S., Craig, J. C., \& Bensmaia, S. J. (2009). Convergence of submodality-specific input onto neurons in primary somatosensory cortex. J Neurophysiol, 102(3), 1843-1853.

Perez-Bellido, A., Barnes, K. A., Crommett, L. E., \& Yau, J. M. (2017). Auditory frequency representations in human somatosensory cortex. Cerebral Cortex. https://doi.org/10.1093/cercor/bhx255

Powers, D. M. (2011). Evaluation: From Precision, Recall and F-Measure to ROC, Informedness, Markness \& Correlation. Journal of Machine Learning Technologies, 2(1), 37-63.

Prsa, M., Morandell, K., Cuenu, G., \& Huber, D. (2019). Feature-selective encoding of substrate vibrations in the forelimb somatosensory cortex. Nature, 567(7748), 384-388. https://doi.org/10.1038/s41586-019-1015-8

Rosenbaum, R., Rubin, J., \& Doiron, B. (2012). Short Term Synaptic Depression Imposes a Frequency Dependent Filter on Synaptic Information Transfer. PLoS Computational Biology, 8(6), e1002557. https://doi.org/10.1371/journal.pcbi.1002557 
631 Saal, H. P., \& Bensmaia, S. J. (2014). Touch is a team effort: interplay of submodalities in cutaneous sensibility. Trends Neurosci, 37(12), 689-697. https://doi.org/10.1016/j.tins.2014.08.012

Saal, H. P., Harvey, M. A., \& Bensmaia, S. J. (2015). Rate and timing of cortical responses driven by separate sensory channels. ELife, 4(DECEMBER2015). https://doi.org/10.7554/eLife.10450

Saal, H. P., Wang, X., \& Bensmaia, S. J. (2016). Importance of spike timing in touch: an analogy with hearing? Current Opinion in Neurobiology, 40, 142-149. https://doi.org/10.1016/j.conb.2016.07.013

Schäfer, K., Blankenburg, F., Kupers, R., Grüner, J. M., Law, I., Lauritzen, M., \& Larsson, H. B. W. (2012). Negative BOLD signal changes in ipsilateral primary somatosensory cortex are associated with perfusion decreases and behavioral evidence for functional inhibition. Neurolmage, 59(4), 3119-3127. https://doi.org/10.1016/j.neuroimage.2011.11.085

Shmuel, A., Yacoub, E., Chaimow, D., Logothetis, N. K., \& Ugurbil, K. (2007). Spatio-temporal pointspread function of functional MRI signal in human gray matter. Neuroimage, 35(2), 539-552.

Talbot, W. H., Darian-Smith, I., Kornhuber, H. H., \& Mountcastle, V. B. (1968). The sense of fluttervibration: comparison of the human capacity with response patterns of mechanoreceptive afferents from the monkey hand. Journal of Neurophysiology, 31, 301-334.

Wang, X. (2007). Neural coding strategies in auditory cortex. Hearing Research, 229, 81-93.

Yau, J. M., Olenczak, J. B., Dammann, J. F., \& Bensmaia, S. J. (2009). Temporal Frequency Channels Are Linked across Audition and Touch. Curr Biol, 19(7), 561-566.

Yau, J. M., Weber, A. I., \& Bensmaia, S. J. (2010). Separate mechanisms for audio-tactile pitch and loudness interactions. Front Psychology, 1(160), doi: 10.3389/fpsyg.2010.00160. 


\title{
Signatures of vibration frequency tuning in human neocortex
}

\author{
Lingyan Wang \& Jeffrey M. Yau
}

Supplementary Material

7 Supplementary figures

3 Supplementary tables 


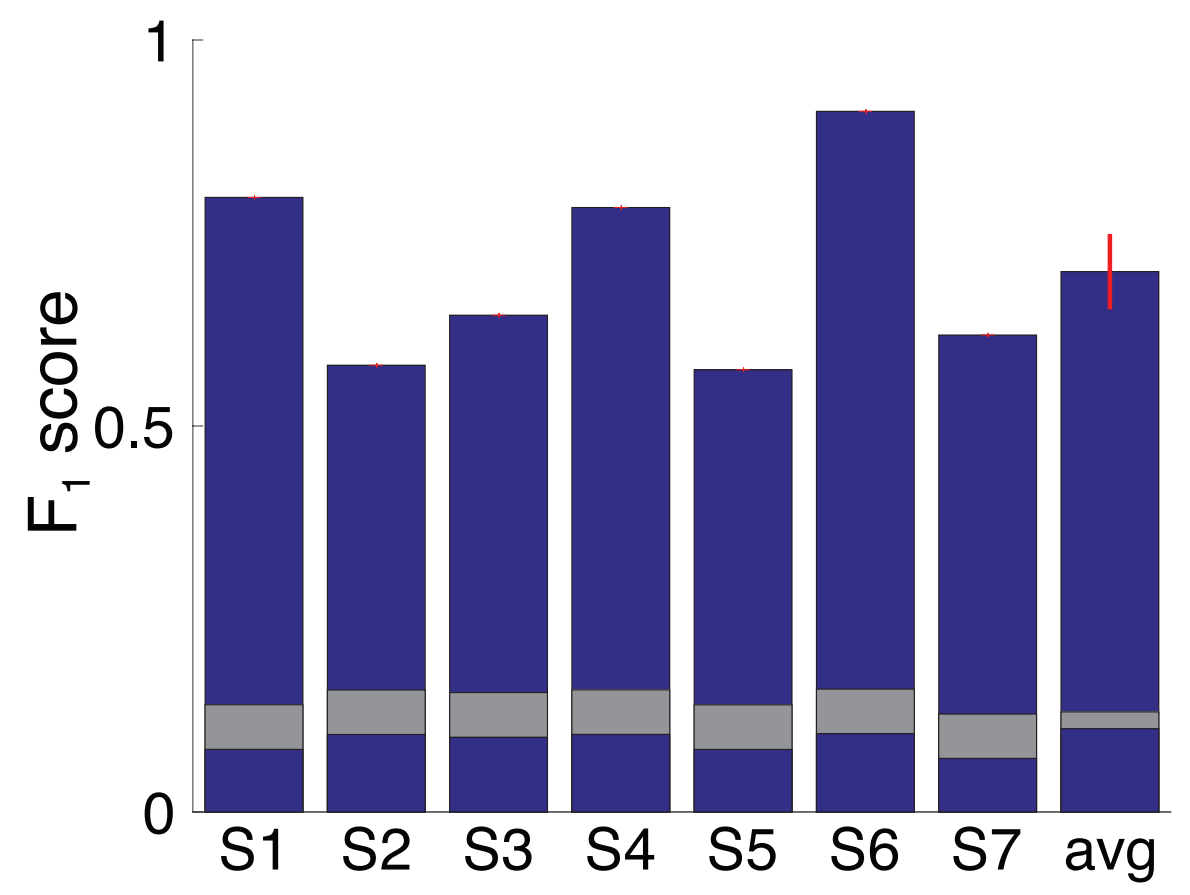

Supplementary Fig. 1. Oddball detection performance. $F_{1}$ score indexes detection performance by accounting for precision and recall ${ }^{1}$. Higher scores indicate better performance. Bars indicate F1 score for each participant and the group-averaged score. Red error bar indicates s.e.m. Gray segments indicates the $F_{1}$ score distributions expected by chance (center = mean score; thickness $=$ standard deviation) given the number of positive responses provided by each participant (Materials and Methods). Performance in each participant far exceeded chance levels. 


\section{a}

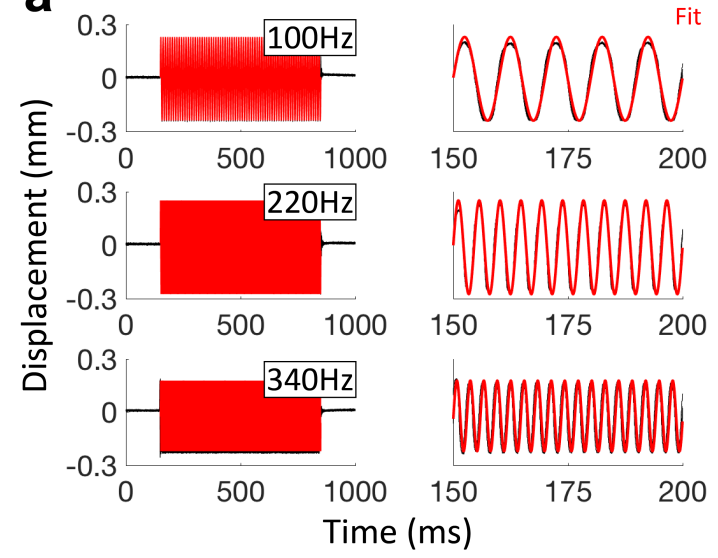

b

\begin{tabular}{|c|c|c|c|}
\hline \multicolumn{2}{|c|}{ Command } & \multicolumn{2}{c|}{ Measured } \\
\hline $\begin{array}{c}\text { Frequency } \\
(\mathrm{Hz})\end{array}$ & Gain & $\begin{array}{c}\text { Frequency } \\
(\mathrm{Hz})\end{array}$ & $\begin{array}{c}\text { Peak-Peak } \\
\text { Displacement }(\mathrm{mm})\end{array}$ \\
\hline 100 & 97.4 & 99.999 & 0.441 \\
\hline 130 & 89.1 & 129.978 & 0.442 \\
\hline 160 & 86.9 & 159.973 & 0.463 \\
\hline 190 & 82.5 & 189.981 & 0.478 \\
\hline 220 & 80 & 219.925 & 0.504 \\
\hline 250 & 78.6 & 249.925 & 0.496 \\
\hline 280 & 76.2 & 279.981 & 0.459 \\
\hline 310 & 73.7 & 310.002 & 0.442 \\
\hline 340 & 71.4 & 339.948 & 0.414 \\
\hline
\end{tabular}

Supplementary Fig. 2. Analysis of measured displacement (a) Unloaded vibration amplitudes were measured outside of MRI environment using a ZX2-LD50 Laser Displacement Sensor (response time $240 \mu \mathrm{s}$; acquired by Power $1401 \mathrm{CED}$ with $40 \mathrm{kHz}$ sampling rate). Displacement profiles for 10 repeats (black) of the 100-, 220-, and 340-Hz stimuli are shown along with fitted sinusoids (red). Waveforms on the right show cycles from a portion of the full measurements. (b) Table indicates command frequencies and gains used to drive stimuli with the Engineering Acoustics, Inc (EAl) controller as well as the measured frequencies and displacements. 

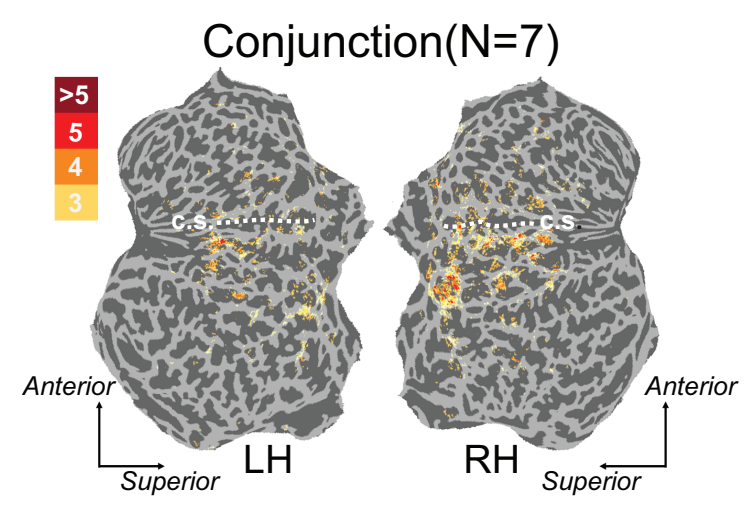

S2

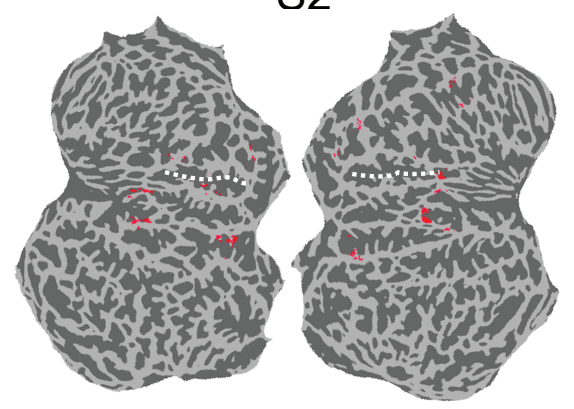

S4
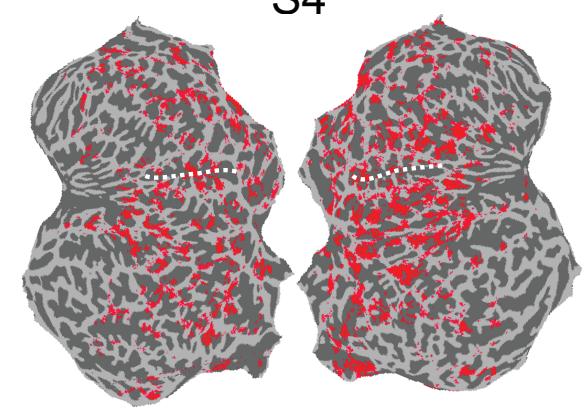

S6
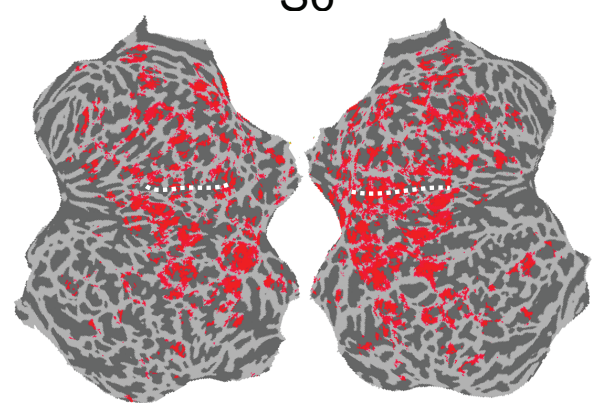

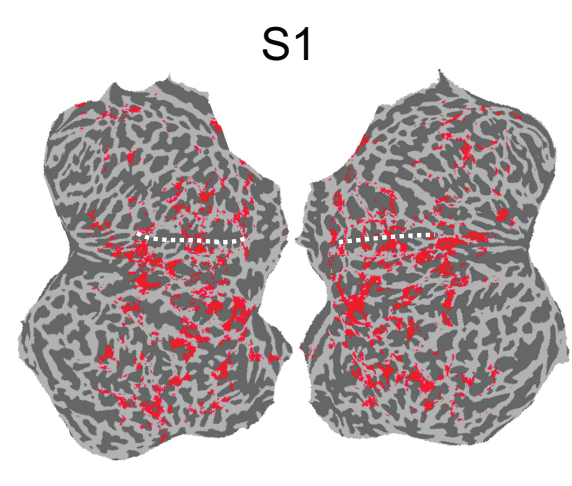

S3

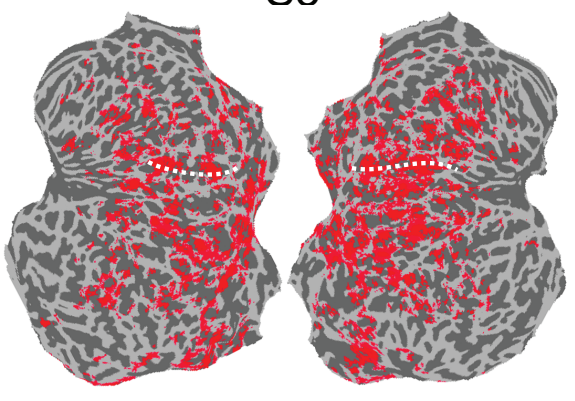

S5

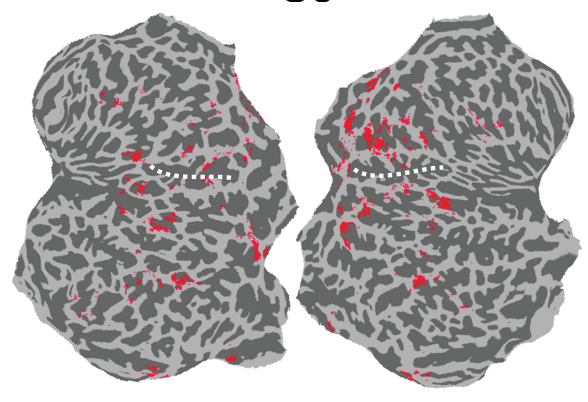

S7
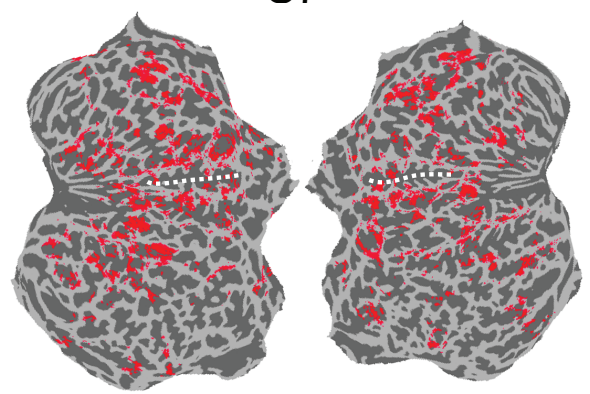

Supplementary Fig. 3. Analysis masks for each participant and group summary mask. Labeled surface nodes indicate significant responses to left or right hand vibrations (FDR corrected $q=$ 0.0001). Conjunction map indicates nodes with significant activations in 3 or more participants. Dashed white lines indicate the central sulcus (c.s.). LH, left hemisphere; $\mathrm{RH}$, right hemisphere 
bioRxiv preprint doi: https://doi.org/10.1101/2021.10.03.462923; this version posted October 27, 2021. The copyright holder for this preprint (which was not certified by peer review) is the author/funder, who has granted bioRxiv a license to display the preprint in perpetuity. It is made available under aCC-BY-NC-ND 4.0 International license.
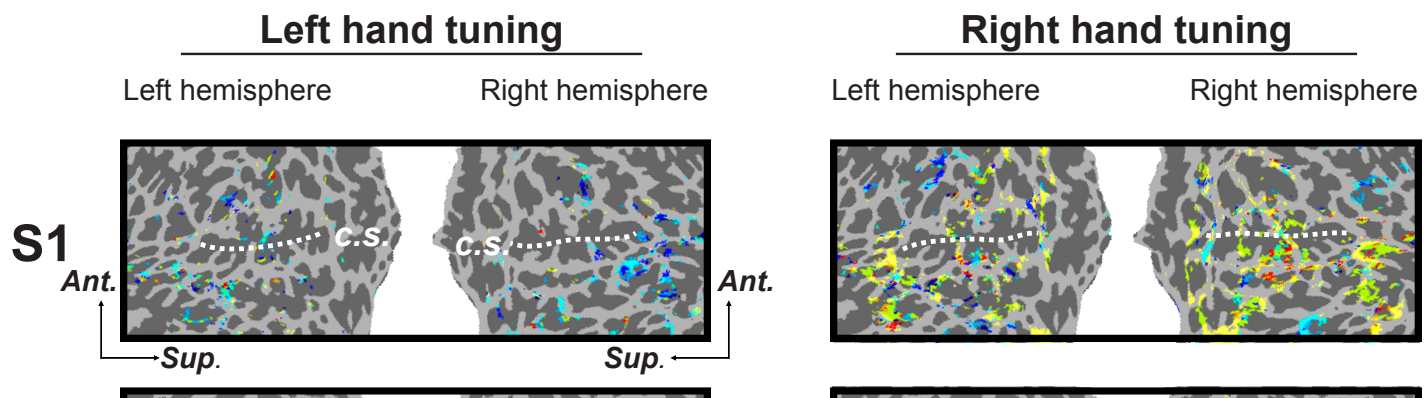

S2
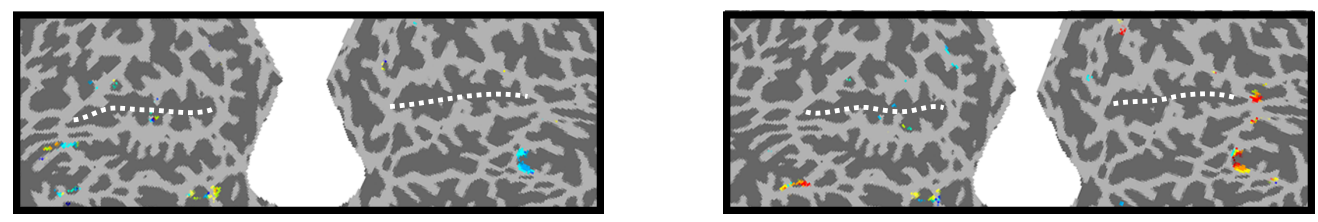

S3
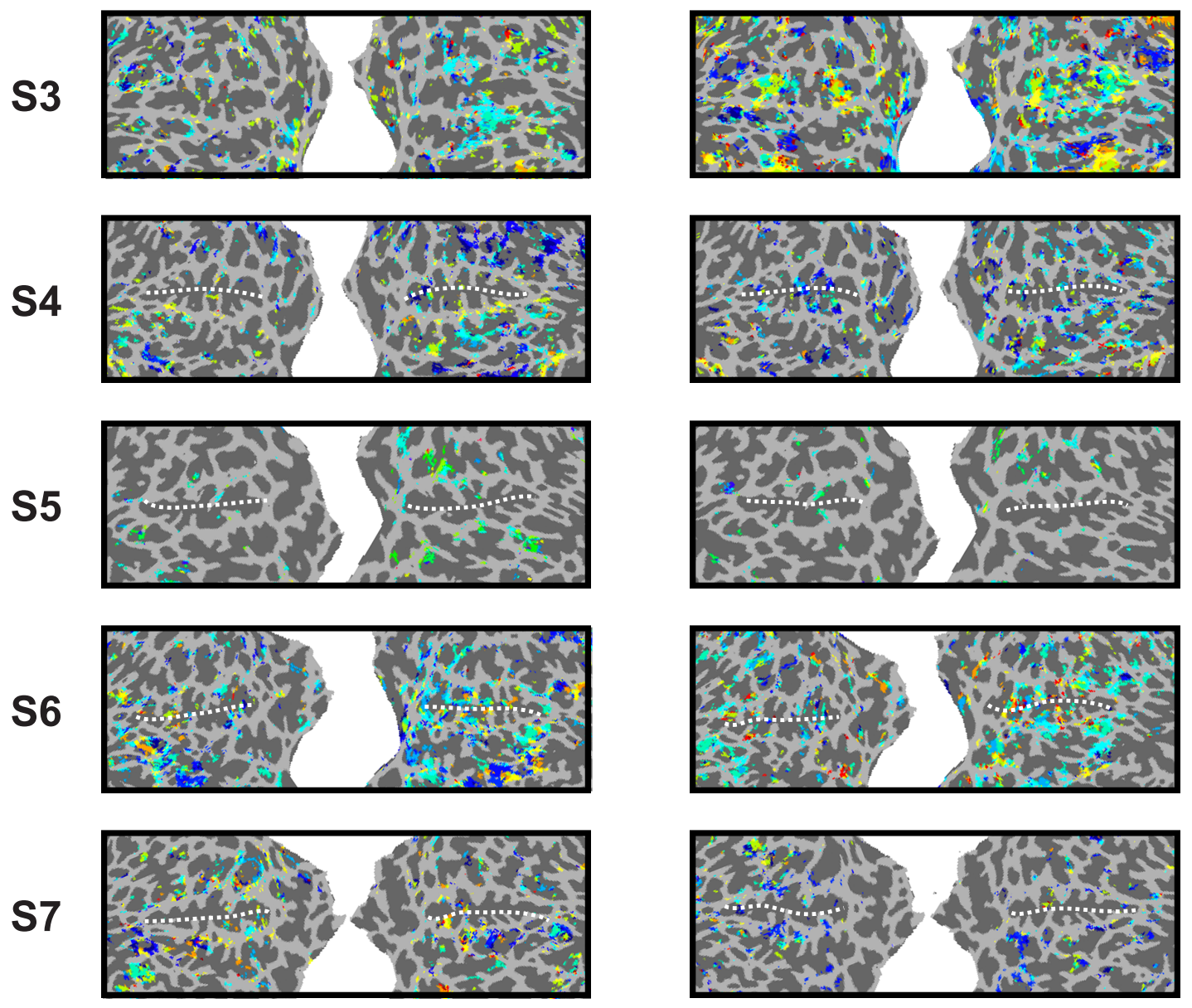

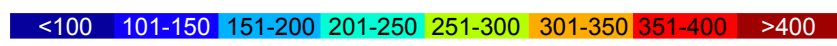
$\mathrm{BF}(\mathrm{Hz})$

Supplementary Fig. 4. Frequency preference maps. Best modulating frequency (BF) for significantly tuned voxels in each participant are projected onto cortical surfaces. Left and right hand tuning is depicted in separate maps. Dashed white lines indicate the central sulcus (c.s.). Ant., anterior; Sup., superior 
a

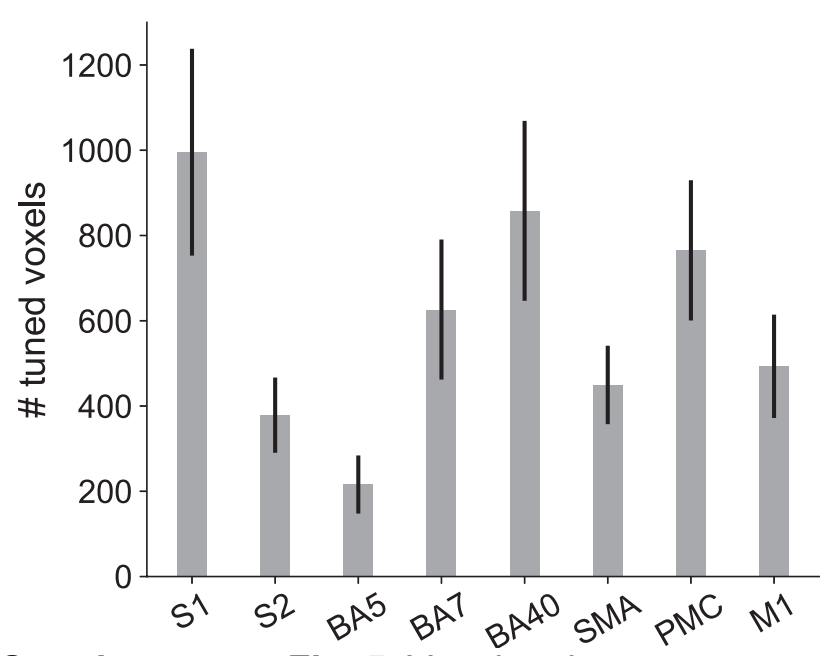

b

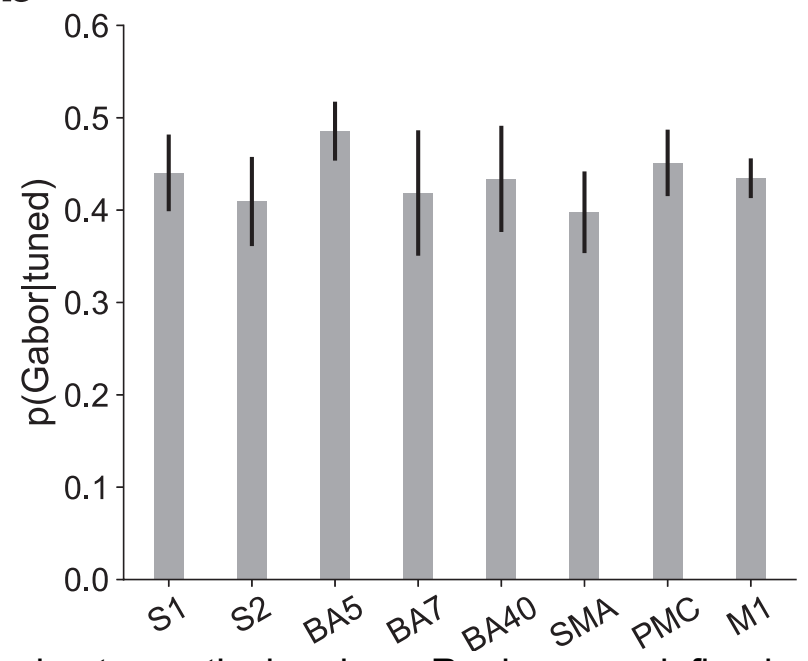

Supplementary Fig. 5. Voxel tuning across sensorimotor cortical regions. Regions are defined using Human Connectome Project parcellations ${ }^{2}$. (a) Number of tuned voxels in sensorimotor regions. (b) Proportion of tuned voxels in each sensorimotor region with response profiles more consistent with Gabor tuning rather than Gaussian tuning according to Akaike Information Criterion. BA, Brodmann area; S1, primary somatosensory cortex; S2, secondary somatosensory cortex; SMA, supplementary motor area; PMC, premotor cortex; M1, primary motor cortex. 

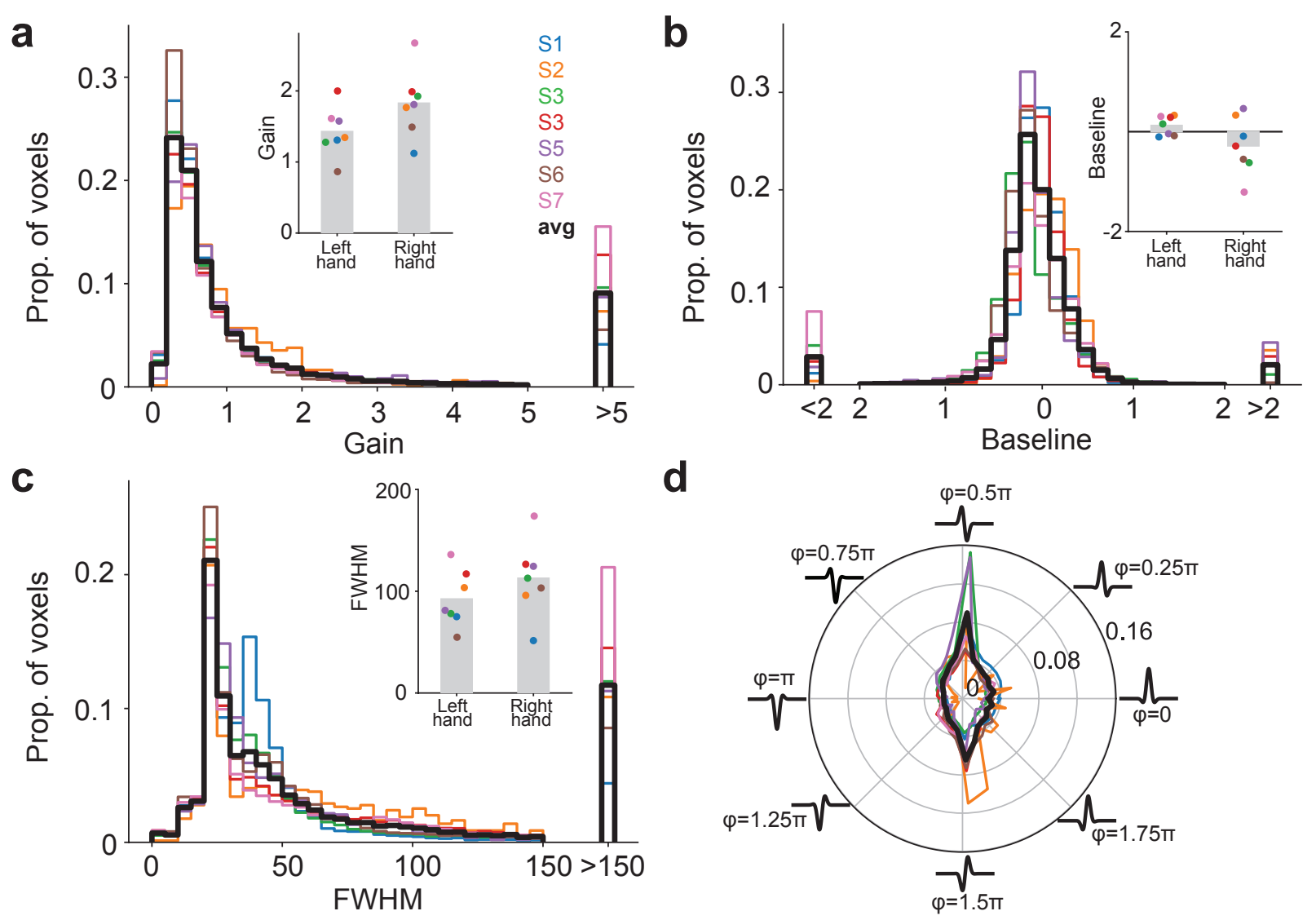

Supplementary Fig. 6. Frequency tuning parameters are highly consistent across participants. (a) Distribution of gain parameters from Gaussian and Gabor functions fitted to tuned voxels. Black traces indicates group average. Inset shows gain parameters sorted according to left hand and right hand tuning functions. Bars indicates group average and dots indicate individual participant averages. Gains were significantly larger for right hand tuning $(t(6)=2.47, P=$ 0.048). (b) Distribution of baseline parameters from Gaussian and Gabor functions fitted to tuned voxels. Conventions as in a. Baseline values did not differ significantly between hands $(t(6)=1.63, P=0.16)$. (c) Distribution of frequency selectivity as indexed by the full-width at half-maximum (FWHM) of the each Gabor or Gaussian tuning function's dominant peak.

Conventions as in a. FWHM did not differ significantly between hands $(t(6)=1.94, P=0.10)$. (d) Distribution of phase parameter values from the Gabor tuning functions. Plotted Gabors indicate canonical profile associated with each phase value. Conventions as in a. Although phase distributions differed between hands (Watson's two-sample test of homogeneity; $U^{2}=0.82-$ 5.66, $P<0.001$ ), there was a consistent pattern for non-uniform phase distributions (Rayleigh test, $P<1 \mathrm{e}-15$ ) with peaks at $\varphi=0.5 \pi$ and $1.5 \pi$. 

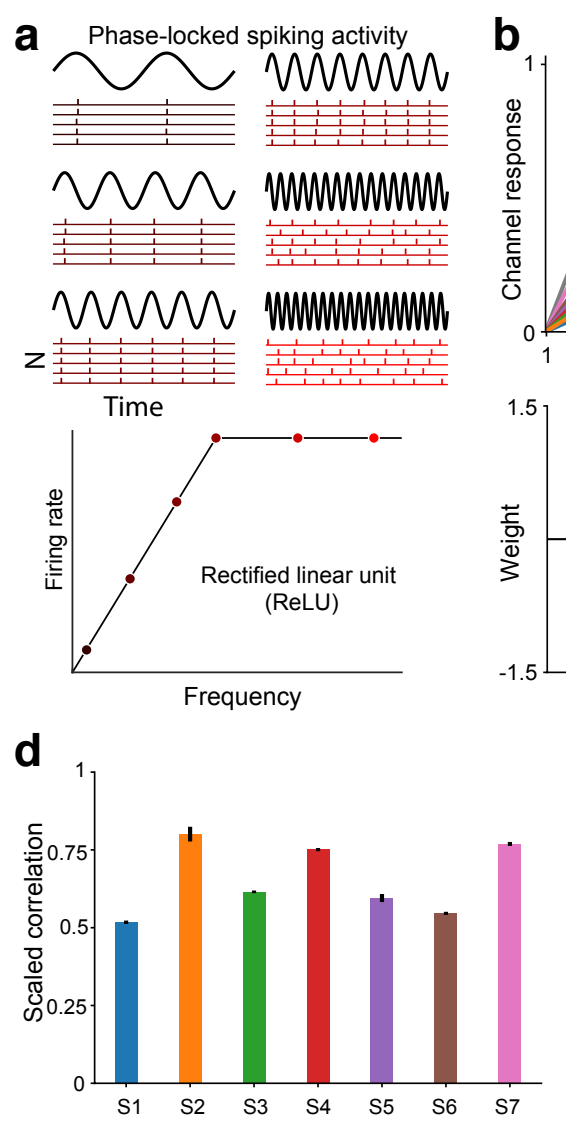
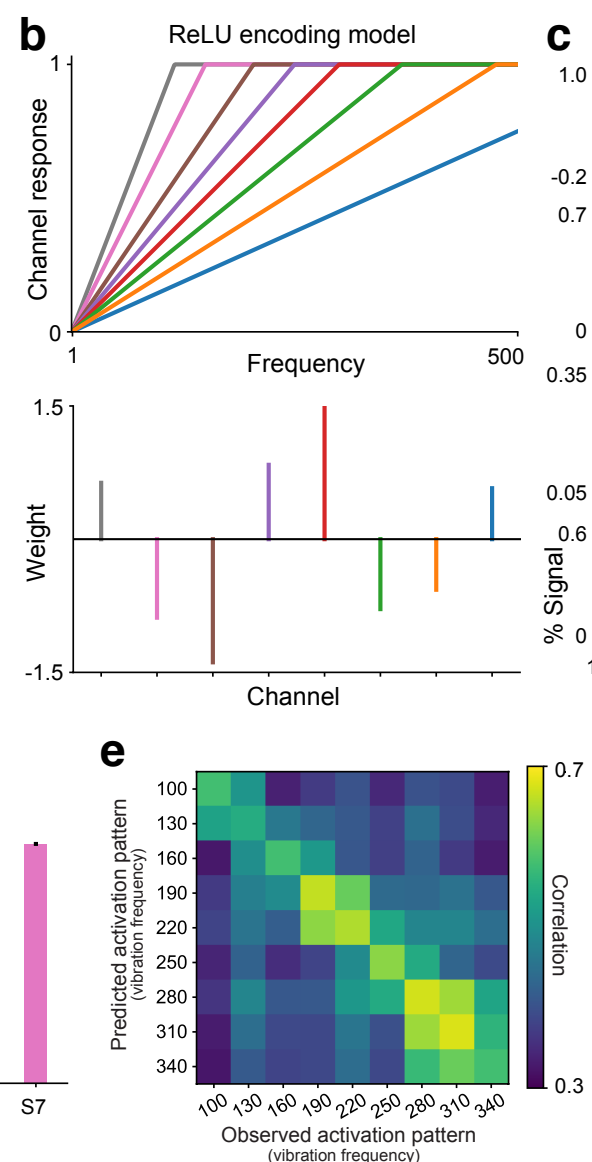
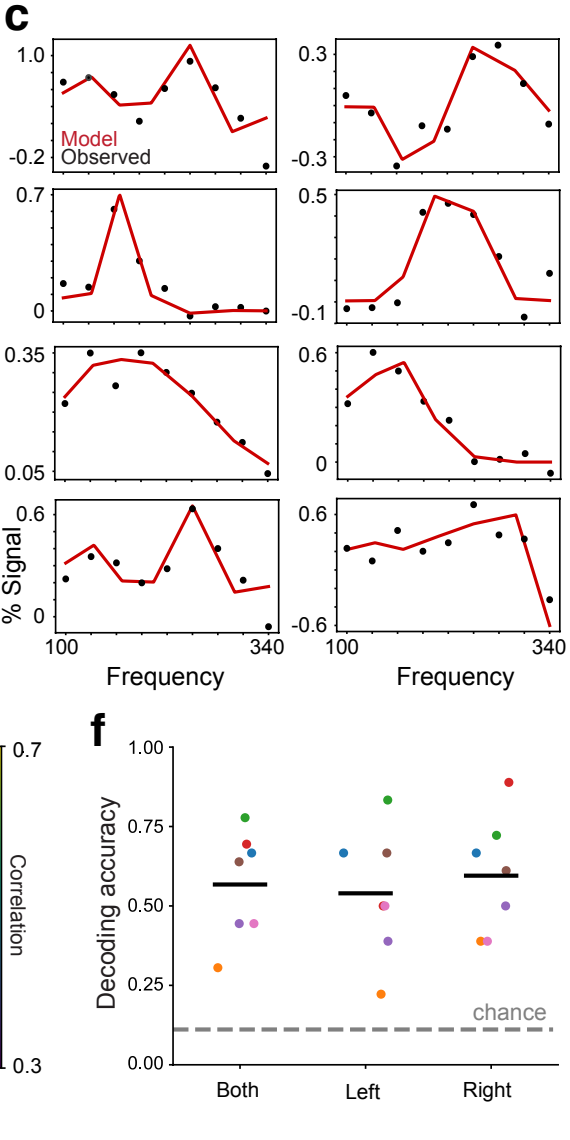

Supplementary Fig. 7. Encoding model based on activity of phase-locking neural populations. (a) Vibration frequency can be encoded in the timing of spiking activity in somatosensory cortical neurons ${ }^{3-5}$. Rasters show idealized activity in neurons whose spikes occur at particular phases of each vibration cycle. At low frequencies, the neurons can fire on every cycle. At high vibration frequencies, phase-locking neurons may skip cycles occurring during their refractory period. Accordingly, the frequency response profile for phase-locking neurons can be described by a low-frequency range over which rates increase monotonically before plateauing at higher frequencies. This profile is captured by a rectified linear activation unit (ReLU). (b) ReLU encoding model assumes that a voxel's response to any given vibration frequency is the weighted sum of the activity in a set of ReLU functions (representing different populations) with different slopes. Slopes are hyperparameters and the weights are estimated in model fitting. Note that the assumption of different slopes implies that the neural populations represented by the ReLU functions are implicitly selective for frequencies. (c) ReLU encoding model (red trace) captures tuned response patterns in example voxels (black dots). (d) Bars indicate voxelaveraged scaled model performance within each participant. The model is trained on one fold of data and tested on a held-out fold. Model performance is the correlation between the model predictions and the test data, normalized by the correlation between the two folds of data (which represent the maximum correlation possible given the noise in the data). Error bars indicate s.e.m. (e) Correlation matrix indicates the similarity between multivoxel activation patterns predicted by the encoding model and the held-out fold. For decoding, the algorithm identifies the model-predicted pattern yielding the highest correlation with an observed pattern to infer the frequency condition. (f) Cross-validated decoding performance for both hands and each hand separately. Black line indicates group averaged accuracy. Colored dots indicate individual participants. Chance performance is $11 \%$. 


\section{Supplementary Table 1 - Number of tuned voxels in the left and right hemispheres}

\begin{tabular}{ccc}
\hline PARTICIPANT & $\begin{array}{c}\text { LEFT } \\
\text { HEMISPHERE }\end{array}$ & $\begin{array}{c}\text { RIGHT } \\
\text { HEMISPHERE }\end{array}$ \\
\hline 1 & 6009 & 6701 \\
2 & 273 & 321 \\
3 & 10357 & 11027 \\
4 & 7668 & 8024 \\
5 & 1423 & 1329 \\
6 & 7974 & 8877 \\
7 & 6088 & 5446
\end{tabular}

Counts indicate the number of tuned voxels in each participant. Vibration-responsive voxels were considered tuned only if they exhibited reliable across-fold correlations $(r>0.2)$ and significant tuning function fits (FDR corrected $q<0.05$ ). A voxel was included in the counts only once regardless of whether it was tuned for both hands. 
Supplementary Table 2 - Number of voxels tuned to the contralateral hand, ipsilateral hand, or both hands

\begin{tabular}{|c|c|c|c|}
\hline PARTICIPANT & CONTRA & IPSI & BOTH \\
\hline 1 & 5348 & 4986 & 2376 \\
\hline 2 & 148 & 248 & 198 \\
\hline 3 & 8684 & 9267 & 3433 \\
\hline 4 & 6640 & 5646 & 3406 \\
\hline 5 & 1167 & 1152 & 433 \\
\hline 6 & 5917 & 6906 & 4028 \\
\hline 7 & 5370 & 4467 & 1697 \\
\hline
\end{tabular}

Counts indicate the number of voxels over the left and right hemispheres in each participant that exhibited tuning only for the contralateral (CONTRA) or the ipsilateral (IPSI) hand. Voxels that were tuned for both hands are indicated in the $3^{\text {rd }}$ column. 


\section{Supplementary Table 3 - Voxel-level frequency preferences}

\begin{tabular}{|c|c|c|c|c|}
\hline PARTICIPANT & BF MEAN & BF MEDIAN & KS STAT & P VALUE \\
\hline 1 & 237 & 267 & 0.99 & $1 e-15$ \\
\hline 2 & 258 & 273 & 0.99 & $1 \mathrm{e}-15$ \\
\hline 3 & 238 & 252 & 0.99 & $1 \mathrm{e}-15$ \\
\hline 4 & 187 & 179 & 0.99 & $1 e-15$ \\
\hline 5 & 202 & 195 & 0.99 & $1 e-15$ \\
\hline 6 & 221 & 216 & 0.99 & $1 \mathrm{e}-15$ \\
\hline 7 & 212 & 238 & 0.99 & $1 e-15$ \\
\hline
\end{tabular}

Values indicate best modulating frequency (BF) statistics in each participant. KS, KolmogorozSmirnov test statistic 


\section{Supplementary References}

1. Powers, D.M. J. Mach. Learn. Technol. 2, 37-63 (2011).

2. Glasser, M.F. et al. Nature 536, 171-178 (2016).

3. Harvey, M.A., Saal, H.P., Dammann 3rd, J.F. \& Bensmaia, S.J. PLoS Biol 11, e1001558 (2013).

4. Mountcastle, V.B., Talbot, W.H., Sakata, H. \& Hyvärinen, J. J. Neurophysiol. 32, 452-484 (1969).

5. Lebedev, M.A. \& Nelson, R.J. Exp. Brain Res. 111, 313-325 (1996). 